\title{
Similarity: Its definition and effect on the visual analysis of complex displays
}

\author{
MARTIN H. SINGER and JOSEPH S. LAPPIN \\ Vanderbilt University, Nashville, Tennessee 37240
}

\begin{abstract}
Contemporary feature models of form perception have typically defined visual similarity in terms of shared (or discordant) sets of points. Two experiments tested the adequacy of this definition. In a same/different task, subjects were required to detect a single "different" form in displays of twa four, or six forms. In separate conditions, the "different" form was produced by various geometrie transformations, where the number of discordant points could be held constant for some of those transformations. The first experiment compared the detectability of three transformations: deletion of an end-of-a-line segment, a break in continuity, and a mirror-image reversal. Reversals were detected most rapidly and accurately, with performance independent of display size. Although breaks and deletions produced the same number of discordant points, breaks were detected more rapidly and accurately. The second experiment tested whether the better detectability of reversals was due to a greater number of discordant points or to changes in the orientation of diagonal lines. The results indicated that entire displays can be rapidly organized (in "parallel") on the basis of line orientations. In general, the experiments suggest that the similarity of forms may depend upon the transformations by which they are related rather than their common features.
\end{abstract}

The speed with which subjects can detect a visual form embedded in a complex display depends on the similarity among the displayed forms. In visual search tasks, where subjects are asked to detect the presence of a specified "target," speed and accuracy of detection improve as the similarity or confusability of the target and "noise" items is decreased, and also improve as the similarity of the various "noise" items is increased (Atkinson, Holmgren, \& Juola, 1969; Estes, 1972). Indeed, when the target and noise items are dissimilar and when the noise items are homogeneous, speed and accuracy are often found to be independent of display size (in contrast to the more typical decline in performance with increasing display size). This has been demonstrated by McIntyre, Fox, and Neale (1970), as well as by Estes (1972).

The proper definition and measure of similarity, however, are uncertain. Two alternative approaches can be identified. One approach characterizes similarity as a result of the analysis of a form's component parts or "features" (Estes, 1972; Reed, 1973; Shiffrin \& Geisler, 1973; Wolford, 1975). The similarity of two forms could thus be measured by the number of common features shared by the two

This research was supported in part by Public Health Service Grant MH 21105. The report was written while the authors were supported by grants from the Spencer Foundation and the Vanderbilt University Research Council. The authors gratefully acknowledge the excellent computer programming assistance of Melanae Schnurr and the creative assistance of Brian Kottas in developing the second experiment. This paper was presented at MPA, 1975. Requests for reprints should be sent to Martin Singer, Department of Psychology, Vanderbilt University, Nashville, Tennessee 37240. forms. Another approach, gestalt in nature, emphasizes the importance of relationships within and between various forms. Accordingly, similarity might be defined by the transformations required to change one form into the other.

Estes' (1972) model provides a detailed example of the feature approach. Estes argues that the effects of similarity can be explained in terms of interactions among visual channels that provide input to feature detectors. Similar items activate overlapping sets of feature detectors. Noise items that are similar to the target can thus be confused with the target. Furthermore, because the input channels have the capability to interact and can prevent the further processing of redundant information, homogeneous noise items tend to inhibit each other and, as a result, increase the salience of the target.

While it is not exactly clear what comprises a "feature" in most feature models, the definition employed by McIntyre et al. (1970) usually seems implicit. That is, "an estimate of the similarity between any pair of letters can be made by conceptually superimposing the pair and counting the number of physical features they have in common" (p. 330). In other words, features appear to be subsets of points and dissimilarity might be measured by the number of discordant points.

Although feature models account for most of the published results on the processing of briefly presented visual displays, Egeth, Atkinson, Gilmore, and Marcus (1973) note exceptions. First, in some visual search tasks where noise items were heterogeneous, reaction time (RT) remained constant across different display sizes (Egeth, Jonides, \& 


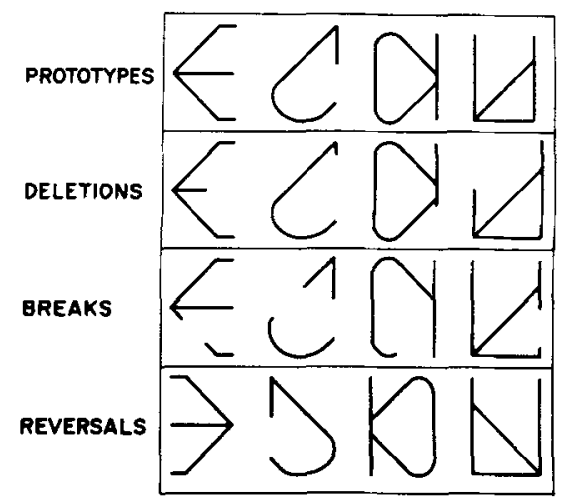

Figure 1. The prototypes and their three respective transforms that were used in Experiment $I$ are displayed above.

Wall, 1972). Secondly, Jonides and Gleitman (1972) demonstrated that the "conceptual category" of target and noise elements can alter the relationship between RT and display size. In that experiment, " 0 "' was the target and noise items were either all letters or all digits. Subjects were told to either detect the letter "oh" or the numeral zero. When the "conceptual category" of the target and noise items differed, RT remained invariant with respect to display size. When target and noise elements shared the same category, however, RT increased as a function of display size. The results are particularly interesting in that target characteristics (e.g., features) remained constant across conditions.

The alternative to the hypothesis that form perception is derived from feature detection, as mentioned earlier, is to suppose that the perceptual system is sensitive to relationships within and among forms. Just as the detectability of a target depends on its similarity with the other displayed forms (Neisser, 1967), it seems likely that the relevant properties of a feature depend upon its context within the whole form. This approach seems compatible with the rationale and results of same/different experiments, in which the subject is asked to determine whether or not a set of forms is identical. A common finding in such a task is that even "same" displays are rapidly detected, independently of display size. Bamber (1969) has suggested the operation of an “identity detector" to account for such results.

A similar interpretation, as well as perhaps a "difference detector," seems indicated by the demonstratons of Beck (1972) and Julesz, Gilbert, Shepp, and Frisch (1973). In those experiments, discrepant subsets of very large displays were rapidly detected when the subset was composed of line orientations different from the rest of the display. Differences in line orientation apparently allowed subjects to quickly organize large displays into "same" and "different" subsets. All of these results suggest the importance of relationships between forms. The work of Julesz et al. represents a thorough investigation of the geometric properties of form that permit rapid detection of contrasting forms, but it is not certain how their results generalize to the perception of smaller displays and to the slower, serial-like processing of individual forms.

The general method of the present experiments was to use the same/different task-where the subject was required to decide whether all of the displayed forms were identical or whether any one was different-as a means for testing the competing definitions of similarity. Independent variables were the particular transformations used to produce a difference in forms and the number of forms in the display. Both speed and accuracy of the "same" vs. "different" responses were used as dependent measures for evaluating the effect of display size on the detectability of a given Iransformation.

The principal question addressed by our first experiment was whether the detectability of a difference in forms is determined by the number of discordant points or, alternatively, whether the detectability of a transformation depends upon its context within the whole form. Specifically, the detectability of three transformations was examined: Deletion of a set of points from the end of a line segment, a break in the continuity of a line segment, and a left-right mirror image reversal of the whole form. As may be seen in Figure 1, the deletion and break transformations produced the same number of discordant points, while the reversal transformation produced a much larger number of discordant points but no change in the overall shape of the form. Thus, a difference in detectability of the deletions and breaks would indicate that the perceptual dissimilarity produced by the removal of a line segment depends upon the position of the line segment within the whole form. Similarly, if the reversal transformations were more difficult to detect than the other transformations, then the perceptual similarity of a form and its reversal would not be based on numbers of discordant points, but instead on more global properties of the overall shape. We anticipated both of these results, indicating the inadequacy of the number of discordant points as a measure of similarity. As it turned out, however, the reversals were much easier to detect than the other transformations, in contrast to earlier experiments using reversal transformations (Gibson, Gibson, Pick, \& Osser, 1962; Lappin, 1971). Moreover, the size of the display had no effect on the speed and accuracy in detecting these transformations. A second experiment was then conducted to determine whether the effectiveness of the reversal transformation resulted from the greater number of discordant points it produced or from a change in the orientation of diagonal line segments. The results of both 
experiments shed light on the influence of visual similarity on the mode of perceptual processing of displays of multiple forms.

\section{EXPERIMENT I}

\section{Method}

Subjects. Nine male graduate students in psychology at Vanderbilt University voluntarily participated in the experiment. The subjects' ages ranged from 22 to 34 years.

Apparatus and stimuli. A PDP-8/f computer generated visual displays on a cathode ray tube (Tektronix 604 with P-15 phosphor). Telegraph keys served as response mechanisms, and a Teletype bell was used to provide feedback. The figures generated by the computer appeared at any of 12 locations around a circular display (after Egeth et al., 1972) with the constraint that the symbols were maximally spaced. A circular array was used to reduce the transfer of reading-related skills and to minimize differences in acuity due to retinal position. The diameter of this circular array subtended a visual angle of $2.6 \mathrm{deg}$; the individual items subtended a visual angle of $31 \times 23 \mathrm{~min}$.

The figures used in this experiment were adapted from the Gibson et al. (1962) experiment. The four figures taken from their study were prototypes from which we derived all of the transforms. Both the prototypes and transforms were constructed within 13 by 9 matrices of dots on the display screen.

Three transforms were constructed from each of the four figures. In one case, a transform was constructed by deleting four or five dots from a line element contained in the prototype. This transformation, however, left the continuity of the prototype intact (a deletion). It was sometimes necessary to remove five dots in this condition to ensure that length of the deleted line was equal to the deleted segment in the second condition. The second transform was formed by removing four dots such that the continuity of the prototype was disrupted (a break). A third transform was a mirror-image reversal around the vertical axis (a reversal). Although the figures were made up of dots, the figures on the display appeared to be composed of continuous lines. Figure 1 illustrates the four prototypes and their respective transforms.

Procedures. The original design of this experiment involved only one group of subjects instructed to make same-different judgments within a rapid deadline. Because the RTs were essentially constant across conditions, error rate was the only dependent measure for this group. The experiment was then replicated using $\mathrm{RT}$ as the dependent measure. A second group of subjects was instructed to concentrate on the accuracy of their responses. Three of the subjects in the original group plus three new subjects participated in this second high-accuracy group. Having three subjects from the first group participate in the second group allowed us to test whether or not any of the obtained effects disappeared with extended practice.

In both instructional groups, the subjects participated in four sessions, the first of which was practice. Each session included three blocks of trials, where each block was devoted to discriminations between the prototype and one of the three transformations. That is, only one of the three transformations occurred in a block of trials. The order of the conditions within each session was counterbalanced across sessions and subjects.

Display sizes were randomly mixed within each block of trials. A block contained 196 trials, and display sizes of two, four, and six occurred with equal probability. The probability of a "same" or "different" display occurring was also equal to .5. Also, given a "different" display, the position of the "different" element was randomly varied and occurred with equal probability.

Care was taken to ensure that the tasks involved samedifferent judgments and were not simply detection tasks. In the deletion condition, as an example, a "different" display could contain all prototypes and one deleted transform or all transforms and one prototype. This technique forced subjects to make comparisons rather than search for a deletion. The same safeguard was employed in the other two conditions.

The display was viewed binocularly from a distance of about $125 \mathrm{~cm}$ in a dimly lit room. The subjects initiated a trial by depressing the two response keys. Immediately, an " $X$ " appeared on the center of the screen, and this was followed $500 \mathrm{msec}$ later by a display containing two, four, or six elements. If the subjects judged all the elements to be similar. they released the left telegraph key. If they detected a different element, they released the right key. If they made the correct decision, a Teletype bell rang. After completing a block of trials, the subjects took a brief rest before beginning the next trial block.

The first group was instructed to respond within a $500-\mathrm{msec}$ deadline. The deadline was signaled by the offset of the display. They were asked to be as accurate as possible within this constraint, but beating the deadline was emphasized. Error rate was the dependent measure for this group, as one intent of the instructions was to keep RT constant across the three conditions.

The other group was asked to respond before a 1.0-sec deadline. It was explained that this deadline afforded enough time to allow very accurate judgments. RT was the dependent measure for this group, as we expected error rate to be consistently low across the different conditions. Responses were not discounted in either group if the subjects exceeded the deadline.

The subjects completed all of their sessions within I week. Approximately 2 weeks elapsed after the first group finished before the second group began.

\section{Results and Discussion}

Data for the error rate group were made conditional upon the responses-i.e., the probability of a same display occurring given a "same" response and the probability of a different display given a "different" response. The intent of this procedure was to eliminate the effects of response biases. When errors are made conditional upon the displays rather than on the responses, then there are opposite trends for the same and different displays, with the error rate decreasing with display size on the "same" trials and increasing on the "different" trials. These trends reflect, in part, an increasing bias for "same" responses with increased display sizes; the improvement for the larger "same" displays disappears when errors are made conditional upon responses. In the RT group, we separated the latencies for "same" and "different" responses. Table 1 summarizes the data for both groups.

The subjects found the deletion condition to be the hardest. In the RT group, it took subjects a longer time to make same-different judgments when a deletion transformation produced any "differences." In the other group, error rate was highest on the deletion condition. Both groups performed better on the break condition. In both of these conditions, however, display size similarity affected subjects' performance.

Subjects in both groups performed best on the reversal conditions. They often reported that the "different" figure seemed to "pop out." They also reported that they could employ a different strategy 
Table 1

Error Rates and Reaction Times for Various Display Sizes and Transformations

\begin{tabular}{|c|c|c|c|c|c|c|c|c|c|c|c|c|}
\hline \multirow{4}{*}{$\begin{array}{l}\text { Group } \\
\text { Response } \\
\text { Display Size }\end{array}$} & \multicolumn{6}{|c|}{ Error Rates* } & \multicolumn{6}{|c|}{$\begin{array}{l}\text { Mean Reaction Time (in msec) } \\
\text { For "same" and "different" response }\end{array}$} \\
\hline & \multicolumn{6}{|c|}{ Error Rate } & \multicolumn{6}{|c|}{ Reaction Time } \\
\hline & \multicolumn{3}{|c|}{$\mathrm{P}(\mathrm{D} \mid \mathrm{s})$} & \multicolumn{3}{|c|}{$P(S \mid d)$} & \multicolumn{3}{|c|}{ "Same" } & \multicolumn{3}{|c|}{ "Different" } \\
\hline & 2 & 4 & 6 & 2 & 4 & 6 & 2 & 4 & 6 & 2 & 4 & 6 \\
\hline $\begin{array}{l}\text { Deletion } \\
\text { Break } \\
\text { Reversal }\end{array}$ & $\begin{array}{l}.27 \\
.19 \\
.15\end{array}$ & $\begin{array}{l}.31 \\
.25 \\
.18\end{array}$ & $\begin{array}{l}.35 \\
.32 \\
.18\end{array}$ & $\begin{array}{l}.22 \\
.21 \\
.15\end{array}$ & $\begin{array}{l}.28 \\
.23 \\
.14\end{array}$ & $\begin{array}{l}.31 \\
.25 \\
.11\end{array}$ & $\begin{array}{l}523 \\
551 \\
476\end{array}$ & $\begin{array}{l}699 \\
609 \\
482\end{array}$ & $\begin{array}{l}733 \\
644 \\
492\end{array}$ & $\begin{array}{l}618 \\
528 \\
468\end{array}$ & $\begin{array}{l}679 \\
605 \\
505\end{array}$ & $\begin{array}{l}723 \\
640 \\
509\end{array}$ \\
\hline
\end{tabular}

*Error rates were made conditional upon responses. Lowercase letters indicate the response type and uppercase letters indicate display type.

in this condition. While the other conditions demanded a figure comparison, in the reversal condition, the subjects claimed that they looked at the entire display. The relationship between RT or error rate and display size may reflect this strategy. In the reversal condition, changes in display size significantly affected the dependent measure. In fact, on the "different" responses in the error-rate group, the accuracy of subjects' responses improved with increases in display size. Their RTs on the reversal condition increased only between Display Sizes 2 and 4 on the "different" latency. Even this increase in RT is much less than the increases in RT on the other two conditions. Figure 2 illustrates these relationships, averaging the data for both types of response.

Two analyses of variance corroborate the above findings. In the first group, where error rate served as the dependent measure, there were significant effects due to the type of transformation, $F(2,10)=$ $21.54, p<.01$, and display size, $F(2,10)=6.27$, $p<.05$, and a significant interaction between display and transformation, $F(4,20)=3.81, p<.05$. The same effects were found for the second group, in which RT was the dependent variable. The analysis disclosed effects due to transformation, $F(2,10)=$ $48.71, p<.01$, display size, $F(2,10)=52.109$, $p<.01$, and the interaction of transformation and display size, $F(4,20)=11.07, p<.01$. There were no differences in the performances of those subjects who performed in both groups and those participating in only one group.

It should be noted that none of these effects is attributable to fluctuations in the irrelevant dependent measure. When error rate was the relevant measure, the mean RTs were $470 \mathrm{msec}$ on the reversal condition, $532 \mathrm{msec}$ on the break condition, and $570 \mathrm{msec}$ on the deletion condition. Likewise, when RT was the relevant measure, the error rates were $7 \%$ on the reversal condition, $10 \%$ on the break condition, and $14 \%$ on the deletion condition.

There are, then, three primary findings in Experiment I. First, differences that break the continuity of a figure are easier for subjects to detect than differences caused by a deletion that leaves the continuity of the figure unaltered. The comparison between the break and deletion conditions indicates the importance of the relationship between a component feature and the whole figure. The difficulty of detecting the deletion or addition of a component
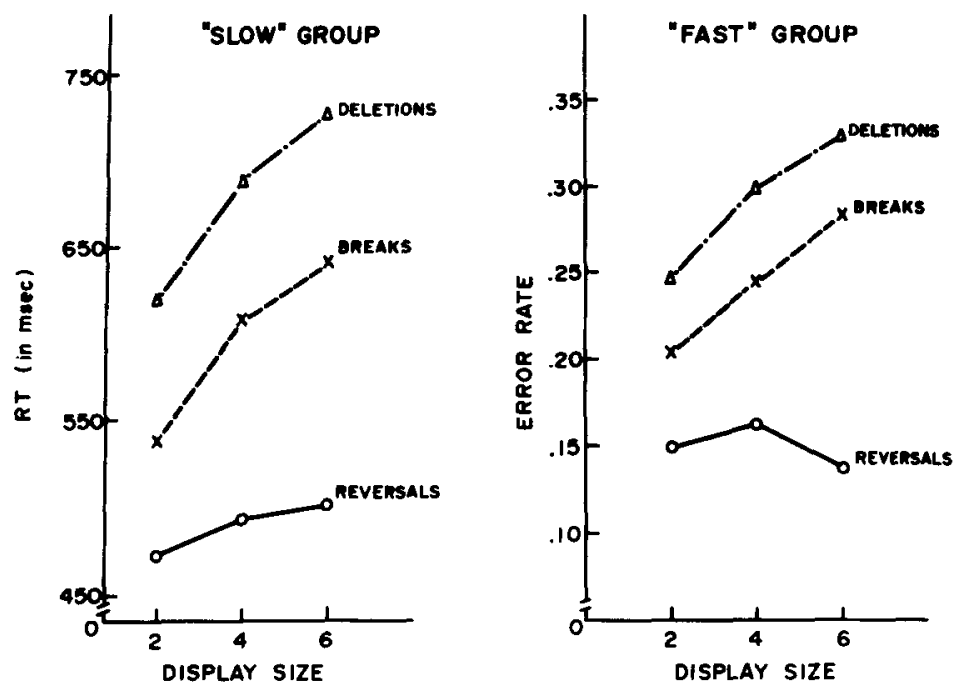

Figure 2. The average of the "same" and "different" responses for the error-rate group and the "same" latency for the RT group are separately illustrated in the two groups. 
line segment varied with its relationship to the whole figure. Second, a reversal transformation produced differences most readily and accurately detected by subjects. This finding is inconsistent with results reported by Gibson et al. (1962) and Lappin (1971). ${ }^{1}$ Finally, increases in display size did not affect subjects' performances on the other two conditions.

We did not expect subjects to perform well on the reversal condition. This result, however, was predictable on the basis of the McIntyre et al. (1970) definition of similarity. That is, if one superimposes a reversed transform upon its prototype, there are more discordant points and fewer "similar" features between them than there are between the other two transforms and the prototypes. Given Estes' (1972) system of inhibitory input channels, the redundant and highly dissimilar "same" figures could increase the salience of the "different" figure.

Subjects' superior performance on the reversal condition is also consistent, though, with a different interpretation. Beck (1972) and Julesz et al. (1973) report that subjects are able to quickly organize visual displays on the basis of line orientation. Julesz et al. (1973) provide examples in which a set of figures which contain line elements that differ in orientation from the line elements in the stimulus context seem to "pop out." He claims that orientation differences between lines allow texture discrimination. There was no such effect when line orientation differences were not present. In Beck's (1972) experiment, subjects could quickly count the number of "different" elements in a visual display when those figures differed from the other display items in regard to line orientation. Subjects were less successful when the "different" figures did not differ in line orientation from the other figures. Subjects in Experiment I could have been quickly organizing the displays on the basis of line orientation rather than attending to an increased number of discordant points between the "same" and the "different" figures.

Since line orientation differences and an increased number of discordant points are confounded in the reversal condition, we cannot decide between the two alternative explanations. A second experiment was carried out that isolated orientation differences as an independent variable.

\section{EXPERIMENT II}

\section{Method}

Subjects. Seven Vanderbilt students, three female and four male, served as subjects. None were paid, but the six undergraduates received course credit for their participation. Ages ranged from 19 to 24 .

Apparatus and Stimuli. The same equipment was used in Experiment II as in Experiment I. The only difference was the figures. Two sets of four figures were developed. These figures

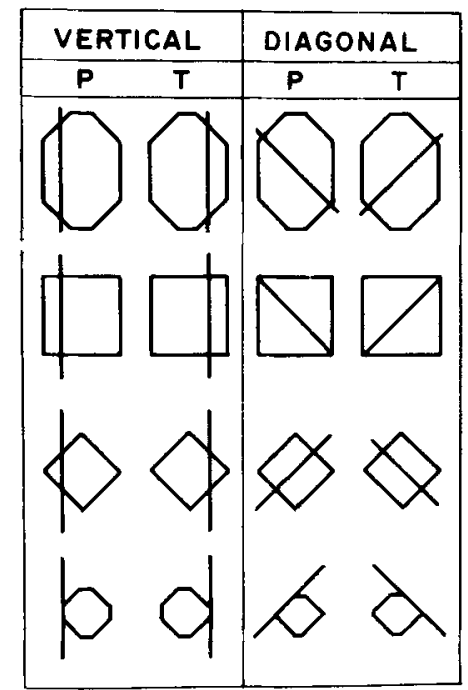

Figure 3. The prototypes and their two respective transforms that were used in Experiment II are displayed above.

were all composed of two basic parts-a bilaterally symmetric polygon plus a vertical or diagonal line. When a reversal transformation was imposed on the diagonal set, it caused line orientation differences between the prototypes and the transforms. In the vertical set, the transformation affected only the left-right serial relation between the vertical line and the symmetric polygon. Because the diagonal lines were purposefully made shorter than the vertical lines, there were sometimes more discordant points between transforms and prototypes in the vertical condition than in the diagonal condition. Figure 3 illustrates the prototypes in the vertical and the diagonal condition and their respective transforms.

Procedures. Again, subjects made same-different judgments in reference to circular displays which contained two, four, and six figures. The vertical and diagonal conditions were presented in different blocks of trials counterbalanced within subjects. Each subject served for one practice and two experimental sessions.

The only major procedural difference from Experiment I was the use of only one dependent measure. Since the two instructional groups provided the same information in Experiment I, Experiment II monitored only error rate in a fast (.5-sec deadline), low-accuracy condition. Subjects again completed the experiment within a week.

\section{Results and Discussion}

Again, the data were made conditional upon the responses. Figure 4 illustrates the difference in performances not only across conditions, but between type of response as well. Only the "same" responses were considered in the data analysis, because their diagnostic value was greater than that of the "different" responses. ${ }^{2}$

An examination of the "same" responses indicates that the addition of figures did not affect error rate in the diagonal condition. It did in the vertical condition, where there were no differences in line orientation between the prototypes and transforms. The subjects apparently organized the displays on the basis of line orientation in the diagonal condition. Judgments in the vertical condition, however, 


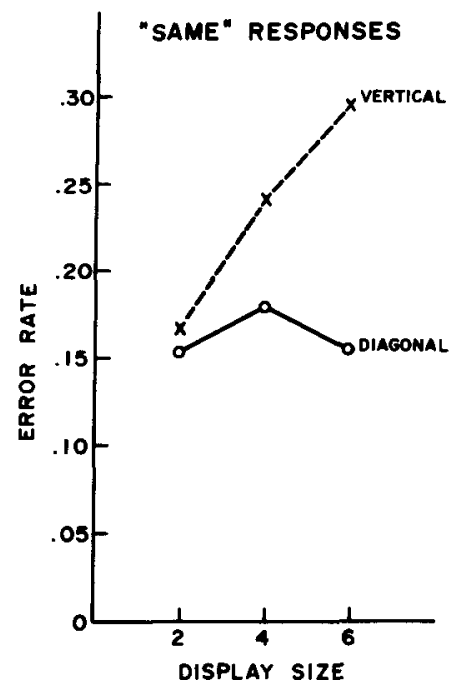

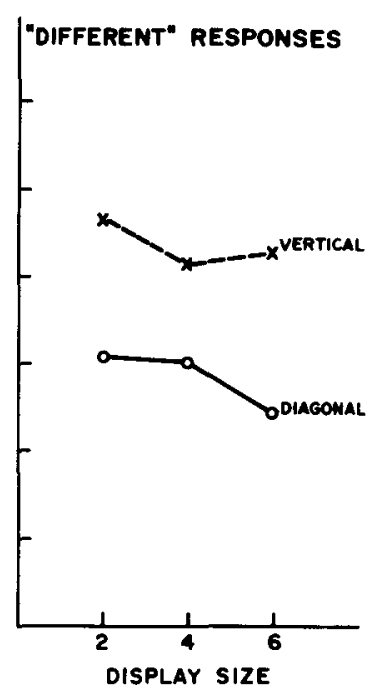

Figure 4. "Same" and "different" responses are separately illustrated for both the vertical and diagonal conditions. seemed to require a figure-by-figure analysis. Julesz et al. (1973) have also demonstrated that differences created by a reversal transformation do not allow rapid texture discrimination unless they also produce differences in line orientation.

The analysis on this data disclosed effects due to condition, $F(1,30)=16.37, p<.025$, and display size, $F(2,30)=5.12, p<.025$. This reflects the differential difficulty of the vertical and diagonal conditions as well as increasing error rates due to increases in display size. Importantly, there was also a significant interaction of display size and condition, $F(2,30)=4.64, p<.025$. This reflects the different relationship between error rate and display size across the two conditions.

In both conditions, the subjects came close to making the deadline. There were differences in RT, and these differences were consistent with the variation in error rate. The mean $\mathrm{RT}$ in the vertical condition was $559 \mathrm{msec}$, and in the diagonal condition, $517 \mathrm{msec}$.

\section{GENERAL DISCUSSION}

The results of these experiments strain the definition of similarity given by contemporary "feature" models of visual. form perception. The results demonstrate that the detectability of a difference between two forms depends upon the context in which that difference occurs: in Experiment I, the detectability of a deleted (or added) line segment depended on the relationship of the line to the whole figure. Secondly, in the diagonal condition of Experiment II, the detectability of a reversal transformation was shown to involve a comparison of line orientations throughout the entire display. In fact, differences in line orientations seemed to be detected by a rapid, parallel processing of all the displayed forms.
If the detection of "features" depends on their context, then, possibly, forms are not really discriminated on the basis of their component features. Given the facilitative effects of redundant context on target detection reported by Estes (1972) and the effects of context obtained in the present study, the concept of separate "features" itself may be questionable. We seem unable, at least, to refer to "features" independently of their context. If models of form perception do discuss "feature differences," it would seem that the description should go beyond reference to collection of discordant points. Again, the reversal condition in Experiments I and II highlight the importance of line orientation in visual similarity.

The effect of various geometric contexts parallel the conceptual category effect reported by Jonides and Gleitman (1972). In that study, conceptual differences allowed subjects to organize an entire display independently of the number of elements comprising the display. In the present study, line orientation differences afforded subjects the same opportunity for organization. The effects of line orientation also coincide with the requirements for texture discrimination discussed by Julesz et al. (1973).

A model accounting for the present data would have to include some explicit mechanism for comparison operations - a mechanism sensitive to relationships among different components of a form and between forms. While it is, of course, possible to append such operations to the feature models discussed earlier, the perceptual analysis of relationships would seem to differ in spirit from the detection of feature in isolation from their context. Perhaps the important issue, however, is whether the detection of relations precedes or follows the detection of the elements. The present experiments were not designed to test this question, but they do demon- 
strate that geometric relations are often rapidly detected.

One reason for the dominance of the concept of "feature" in research and theory on form perception may be the absence of a clear alternative conception. In this regard, the concept of "transformation" might be considered as an alternative. This alternative has experimental support from Pick (1965), who showed that children were able to transfer knowledge about the effect of transformations from one set of prototypes to another. They did not, however, transfer information about specific feature differences. It is possible that a "transformation" represents a general concept which may include the concept of "feature."

An apparent advantage in defining similarity in terms of common features is that such a definition lends itself naturally to measures of similarity. Defining similarity by transformations, however, would seem to deprive the concept of a rationale for measurement: How much dissimilarity is generated by one transformation relative to another? The step backwards is more apparent than real, however. If "features" do not have an a priori definition by independent operations, then similarity is measurable only after the observation of its effects. If similarity should be considered as based on transformations, then the present experiments contribute some initial evidence about the effective similarity associated with various transformations. We have not yet, however, subjected the concept of "transformation" to the same tests for context independence that were employed in these experiments to test the validity of "features."

\section{REFERENCE NOTE}

1. Singer. M. H.. Allen. T. A.. \& Lappin. J. S. Differential abilitics of good and dislexic readers to discriminate visual and auditon sequence's. Paper presented at the meeting of the Southeastern Conference on Linguistics, Nashville, March, 1975.

\section{REFERENCES}

Atrinson, R. C.. Holmgren, J. E., \& Juola, J. F. Processing time as influenced by the number of elements in visual display. Perception \& Psychophvsics, 1969, 6, 321-326.

BAMBER, D. Reaction times and error rates for "same"." different" judgments of multidimensional stimuli. Perception \& Psuchophysics. 1969. 6. 169-174.

BECK. J. Similarity grouping and peripheral discriminability under uncertainty. American Journal of Psychology, 1972, 85, $1-20$.

Egeth. H., Atkinson. J., Gilmore, G., \& Marcus, N. Factors affecting mode in visual search. Perception \& Psychophysics. 1973. 13, 394-402.
Egeth. H., Jonides. J.. \& Wali. S. Parallel processing of multitement displays. Cognitive Psychology. 1972, 3. 674-698.

Estes. W. K. Interactions of signal and background variables in visual processing. Perception \& Psychophysics, 1972, 12. 278-287.

Gibson. E. J., Gibson, J. J., Pick, A. D., \& Osser. H. A developmental study on the discrimination of letter-like forms. Jourial of Comparative and Physiological Psychology. 1962, 55. 897-906.

Jonides. J.. \& Gleitman, H. A conceptual category effect in visual search: 0 as letter or as digit. Perception \& Psychophysics. 1972. 12. 457-460.

Julesz. B.. Gilbert, E. N., Shepp, L. A., \& Frisch/ H. L. Inability of humans to discriminate between visual extures that agree in second-order statistics--revisited. Perçeption. 1973, 2. 391-405.

LAPPIN. J. S. Transformation-invariant cues in the recognition of simple visual patterns. Perception \& Psychophysics. 1971. 10. $367-370$.

McIntyre, C.. Fox, R., \& Neale. J. Effects of noise similarity and redundancy on the information processed from brief visual displays. Perception \& Psychophysics. 1970, 7. 328-332.

Neisser U. Cognitive Psychology. New York: Appleton-CenturyCrofts. 1967.

Pick, A. D. Improvement of visual and tactual form discrimination. Journal of Experimental Psychology, 1965, 69. 331-339.

REED, S. K. Psychological processes in pattern recognition. New York: Academic Press. 1973.

Shiffrin. R. M.. \& Geisler. W. S. Visual recognition in a theory of information processing. In R. L. Solso (Ed.). Contemporary issues in cognitive psychology: The Loyola Symposium. Washington, D.C: V. H. Winston, 1973.

Wolford. G. Perturbation model for letter identification. Psichologital Revien. 1975, 82, 184-199.

\section{NOTES}

1. The Gibson et al. (1962) experiment was done with children, and that might account for the difference in our results. It should be mentioned, however, that in another experiment we had normal and dyslexic children perform these tasks. We again found that all of these children performed best on the reversal condition (Singer, Allen, \& Lappin, Note 1). The reason for the discrepancy has to do in part with the instructions and structure of the task. A full discussion of this difference is, however, irrelevant to the present experiments.

2. Even if subjects were scanning displays serially, the errors on "different" responses would not necessarily increase with display size: If all the displayed items were the same, then it is not clear why larger displays should have an increased tendency to appear "different." Moreover, the effect of display size should be smaller for different than for same displays, since the former require scanning of fewer items on the average in order to determine the correct response. If subjects were scanning the displays serially, then the error rate for "same" responses should increase with display size whatever the rate of scanning (if it were not sufficient to prevent errors) and whatever the response bias. The error rate for "same" responses thus has more diagnostic value than that for "different" responses.

(Received for publication August 11, 1975; revision accepted February 6, 1976.) 